\title{
Fractional Odd-Dimensional Mechanics
}

\section{Ali Khalili Golmankhaneh, ${ }^{1}$ Alireza Khalili Golmankhaneh, ${ }^{2}$ Dumitru Baleanu, ${ }^{3,4}$ and Mihaela Cristina Baleanu ${ }^{5,6}$}

\author{
${ }^{1}$ Department of Physics, Islamic Azad University, Mahabad Branch, 591393-3137 Mahabad, Iran \\ ${ }^{2}$ Department of Physics, Islamic Azad University, Urmia Branch, P.O. Box 969, Uromiyeh, Iran \\ ${ }^{3}$ Department of Mathematics and Computer Science, Cankaya University, 06530 Ankara, Turkey \\ ${ }^{4}$ Institute of Space Sciences, P.O. Box, MG-23, 76900 Magurele-Bucharest, Romania \\ ${ }^{5}$ Faculty of Physics, University of Bucharest, 76900 Bucharest, Romania \\ ${ }^{6}$ National Mihail Sadoveanu High school, District 2, Bucharest, Romania
}

Correspondence should be addressed to Dumitru Baleanu, dumitru@cankaya.edu.tr

Received 28 August 2010; Accepted 26 October 2010

Academic Editor: J. J. Trujillo

Copyright (c) 2011 Ali Khalili Golmankhaneh et al. This is an open access article distributed under the Creative Commons Attribution License, which permits unrestricted use, distribution, and reproduction in any medium, provided the original work is properly cited.

\begin{abstract}
The classical Nambu mechanics is generalized to involve fractional derivatives using two different methods. The first method is based on the definition of fractional exterior derivative and the second one is based on extending the standard velocities to the fractional ones. Fractional Nambu mechanics may be used for nonintegrable systems with memory. Further, Lagrangian which is generate fractional Nambu equations is defined.
\end{abstract}

\section{Introduction}

Derivatives and integrals of fractional-order have found many applications in recent studies in mechanics and physics, for example, in chaotic dynamics, quantum mechanics, plasma physics, anomalous diffusion, and so many fields of physics [1-12]. Fractional mechanics describes both conservative and nonconservative systems $[13,14]$. In mechanics, Riewe has shown that Lagrangian involving fractional time derivatives leads to equation of motion with nonconservative classical derivatives such as friction $[13,14]$. Motivated by this approach many researchers have explored this area giving new insight into this problem [15-37]. Agrawal has presented fractional Euler-Lagrangian equation involving Riemann-Liouville derivatives [16, 17]. Further fractional single and multi-time Hamiltonian formulation has been developed by Baleanu and coworkers [38].

In 1973, Nambu generalized Hamiltonian mechanics which is called now Nambu mechanics. This formalism is shown that provide a suitable framework for the odd 
dimensional phase space and nonintegrable systems [39-43]. By this motivation the authors have fractionalized this formalism [21].

In this work two methods are introduced for fractionalizing of Nambu mechanics. The first method is based on the definition of fractional exterior derivative and fractional forms. The second methods is based on fractionalizing of classical velocity. The resulted equations using these methods may use for complex memorial systems.

This paper is organized as follows.

Section 2 is devoted to a brief review of the fractional derivative definitions and fractional forms. Section 3 contains the classical Nambu mechanics. Section 4 deals with fractionalizing Hamiltonian mechanics using fractional differential forms. Using two different methods in Section 5 the Nambu mechanics has been fractionalized. In Section 6 is defined a Lagrangian which its variation gives the fractional Hamiltonian equations. In Section 7 we present our conclusions.

\section{Basic Tools}

The following subsections contain all mathematical tools used in this manuscript.

\subsection{Fractional Derivatives}

In this section it is briefly presented the definition of the left and the right fractional derivatives of Riemann-Liouville as well as Caputo [6, 7]. The left Riemann-Liouville fractional derivative is defined as

$$
{ }_{a} D_{t}^{\alpha} f(t)=\frac{1}{\Gamma(n-\alpha)}\left(\frac{d}{d t}\right)^{n} \int_{a}^{t} \frac{f(\tau)}{(t-\tau)^{\alpha+1-n}} d \tau
$$

and the right Riemann-Liouville fractional derivative,

$$
{ }_{t} D_{b}^{\alpha} f(t)=\frac{1}{\Gamma(n-\alpha)}\left(-\frac{d}{d t}\right)^{n} \int_{t}^{b} \frac{f(\tau)}{(\tau-t)^{\alpha+1-n}} d \tau
$$

where the order $\alpha$ fulfills $n-1 \leq \alpha<n$ and $\Gamma$ represent the gamma function. An alternative definition of Riemann-Liouville fractional derivative called Caputo derivative that introduced by Caputo in 1967. The left Caputo derivative defined as

$$
{ }_{a}^{C} D_{t}^{\alpha} f(t)=\frac{1}{\Gamma(n-\alpha)} \int_{a}^{t}(t-\tau)^{n-\alpha-1}\left(\frac{d}{d \tau}\right)^{n} f(\tau) d \tau,
$$

and the right Caputo fractional derivative

$$
{ }_{t}^{C} D_{b}^{\alpha} f(t)=\frac{1}{\Gamma(n-\alpha)} \int_{t}^{b}(\tau-t)^{n-\alpha-1}\left(-\frac{d}{d \tau}\right)^{n} f(\tau) d \tau
$$


where the order $\alpha$ satisfies $n-1 \leq \alpha<n$. The Riemann-Liouville derivative of constant isn't zero, but the Caputo derivative of a constant is zero.

\subsection{Fractional Forms}

The calculus of classical differential forms is a powerful tool in applied mathematics. There are so many books that give a clear introduction to this field [44]. In calculus when a new function appears in the scene, it is natural to ask what its derivative is. Similarly with form, it is reasonable to ask what its exterior derivative is. For example a 1-form, integer-order one, can be shown as follows:

$$
\omega=\sum_{i=1}^{n} a_{i} d x_{i}
$$

The classical exterior derivative is defined as

$$
d=d x_{i} \frac{\partial}{\partial x_{i}}
$$

In $[45,46]$ the author generalizes the definition of integer-order vector spaces form to fractional-order one, and denotes it by $F(v, m, n)$. In this notation $v$ is the order of differential form, $m$ the number of coordinate differential appearing in the basis elements, $n$ the number of coordinates. For instance (2.5) is an element of $F(1,1, n)$.

\section{The Definition of Fractional Exterior Derivative}

If the partial derivative in the definition of the classical exterior derivative, is replaced by the fractional-order, definition of fractional exterior derivative is obtained,

$$
d^{v}=\sum_{i=1}^{n} d x_{i}^{v} D_{x_{i}}^{v}
$$

where ${ }_{0} D_{x}^{v}$ is left fractional derivative. Let $\sigma \in F(v, 1, n)$,

$$
\sigma=\sum_{i=1}^{n} \sigma_{i} d x_{i}^{v}
$$

and consider its fractional exterior derivative

$$
d^{v} \sigma=\sum_{i=1}^{n} d^{v}\left(\sigma_{i} d x_{i}^{v}\right)
$$


Using the product rule of exterior fractional derivative,

$$
d^{v} \sigma=\sum_{i=1}^{n} \sum_{j=1}^{n}{ }_{0} D_{x^{j}}^{v} \sigma_{i} d x_{j}^{v} \wedge d x_{i}^{v} .
$$

If $d^{v} \sigma=0$, then

$$
{ }_{0} D_{x^{j}}^{v} \sigma_{i}-{ }_{0} D_{x^{i}}^{v} \sigma_{j}=0
$$

Note that in the following equations, the $\wedge$ sign is omitted between the differential forms.

\section{Nambu Mechanics}

Consider the Hamilton equations

$$
\begin{gathered}
\dot{q}=\frac{\partial H}{\partial p} \\
\dot{p}=-\frac{\partial H}{\partial p} .
\end{gathered}
$$

In another notation

$$
\begin{aligned}
& \dot{q}=\frac{\partial q}{\partial q} \frac{\partial H}{\partial p}-\frac{\partial H}{\partial q} \frac{\partial q}{\partial p}=\frac{\partial(q, H)}{\partial(q, p)} \\
& \dot{p}=\frac{\partial p}{\partial q} \frac{\partial H}{\partial p}-\frac{\partial H}{\partial q} \frac{\partial p}{\partial p}=\frac{\partial(p, H)}{\partial(q, p)} .
\end{aligned}
$$

Nambu generalized these equations to triplet $p, q, r$ dynamical variables with two Hamiltonians $H_{1}, H_{2}$ as follows:

$$
\dot{q}=\left(\begin{array}{ccc}
\frac{\partial q}{\partial q} & 0 & 0 \\
\frac{\partial H_{1}}{\partial q} & \frac{\partial H_{1}}{\partial p} & \frac{\partial H_{1}}{\partial r} \\
\frac{\partial H_{2}}{\partial q} & \frac{\partial H_{2}}{\partial p} & \frac{\partial H_{2}}{\partial r}
\end{array}\right)=\frac{\partial\left(q, H_{1}, H_{2}\right)}{\partial(q, p, r)}
$$




$$
\begin{gathered}
\dot{p}=\left(\begin{array}{ccc}
0 & \frac{\partial p}{\partial p} & 0 \\
\frac{\partial H_{1}}{\partial q} & \frac{\partial H_{1}}{\partial p} & \frac{\partial H_{1}}{\partial r} \\
\frac{\partial H_{2}}{\partial q} & \frac{\partial H_{2}}{\partial p} & \frac{\partial H_{2}}{\partial r}
\end{array}\right)=\frac{\partial\left(q, H_{1}, H_{2}\right)}{\partial(q, p, r)} \\
\dot{r}=\left(\begin{array}{ccc}
0 & 0 & \frac{\partial r}{\partial r} \\
\frac{\partial H_{1}}{\partial q} & \frac{\partial H_{1}}{\partial p} & \frac{\partial H_{1}}{\partial r} \\
\frac{\partial H_{2}}{\partial q} & \frac{\partial H_{2}}{\partial p} & \frac{\partial H_{2}}{\partial r}
\end{array}\right)=\frac{\partial\left(q, H_{1}, H_{2}\right)}{\partial(q, p, r)} .
\end{gathered}
$$

Considering these equations in terms of differential from, take the following 1-form:

$$
\Omega^{(1)}=p d q-H(p, q) d t
$$

The exterior derivative of $\Omega^{(1)}$ is

$$
d \Omega^{(1)}=\left(d p+\frac{\partial H}{\partial q} d t\right) \wedge\left(d q-\frac{\partial H}{\partial p} d t\right)
$$

The Pfaffian equations is obtained, and then the Hamiltonian equations are resulted.

Now in terms of differential forms the Nambu mechanics is obtained using the following form:

$$
\Omega^{(2)}=q d p \wedge d r-H_{1} d H_{2} \wedge d t
$$

The exterior derivative of this form is as follows:

$$
d \Omega^{(2)}=\left(d q-\frac{\partial\left(H_{1}, H_{2}\right)}{\partial(p, r)} d t\right) \wedge\left(d p-\frac{\partial\left(H_{1}, H_{2}\right)}{\partial(r, q)} d t\right) \wedge\left(d r-\frac{\partial\left(H_{1}, H_{2}\right)}{\partial(q, p)} d t\right)=\theta \wedge \rho \wedge \sigma,
$$


where

$$
\begin{aligned}
& \theta=d q-\frac{\partial\left(H_{1}, H_{2}\right)}{\partial(p, r)} d t \\
& \rho=d p-\frac{\partial\left(H_{1}, H_{2}\right)}{\partial(r, q)} d t \\
& \theta=d r-\frac{\partial\left(H_{1}, H_{2}\right)}{\partial(q, p)} d t
\end{aligned}
$$

Now the Paffian equations. Equating them with zero we lead to Nambu mechanics equations.

\section{Fractional Hamilton's Equations}

The fractional generalization of (3.4) can be defined by

$$
\Omega_{\alpha}^{(1)}=p(d q)^{\alpha}-H(p, q)(d t)^{\alpha} .
$$

The fractional exterior derivative of this form is as follows:

$$
d^{\alpha}\left(\Omega_{\alpha}^{(1)}\right)=d^{\alpha}(p) \wedge(d q)^{\alpha}-d^{\alpha} H(p, q) \wedge(d t)^{\alpha}
$$

Taking

$$
\begin{gathered}
d^{\alpha} p=\frac{p^{1-\alpha}}{\Gamma(2-\alpha)}(d p)^{\alpha}, \\
d^{\alpha} H=\frac{\partial^{\alpha} H}{\partial p^{\alpha}}(d p)^{\alpha}+\frac{\partial^{\alpha} H}{\partial q^{\alpha}}(d q)^{\alpha},
\end{gathered}
$$

we have

$$
\begin{aligned}
d^{\alpha}\left(\Omega_{\alpha}^{(1)}\right) & =\frac{p^{\alpha-1}}{\Gamma(2-\alpha)}(d p)^{\alpha} \wedge(d q)^{\alpha}-\frac{\partial^{\alpha} H}{\partial p^{\alpha}}(d p)^{\alpha} \wedge(d t)^{\alpha}+\frac{\partial^{\alpha} H}{\partial q^{\alpha}}(d q)^{\alpha} \wedge(d t)^{\alpha} \\
& =\left(\frac{p^{1-\alpha}}{\Gamma(2-\alpha)}(d p)^{\alpha}-\frac{\partial^{\alpha} H}{\partial q^{\alpha}}(d t)^{\alpha}\right) \wedge\left((d q)^{\alpha}-\frac{\Gamma(2-\alpha)}{p^{1-\alpha}} \frac{\partial^{\alpha} H}{\partial p^{\alpha}}(d t)^{\alpha}\right)
\end{aligned}
$$

The fractional Pfaffian equations are as follows:

$$
\begin{aligned}
& \theta_{\alpha}=\frac{p^{1-\alpha}}{\Gamma(2-\alpha)}(d p)^{\alpha}-\frac{\partial^{\alpha} H}{\partial q^{\alpha}}(d t)^{\alpha}, \\
& \rho_{\alpha}=(d p)^{\alpha}-\frac{\Gamma(2-\alpha)}{p^{1-\alpha}} \frac{\partial^{\alpha} H}{\partial p^{\alpha}}(d t)^{\alpha} .
\end{aligned}
$$


The fractional Hamiltonian equations is resulted

$$
\begin{aligned}
& \frac{p^{1-\alpha}}{\Gamma(2-\alpha)}(d p)^{\alpha}=\frac{\partial^{\alpha} H}{\partial q^{\alpha}}(d t)^{\alpha}, \\
& (d q)^{\alpha}=\frac{\Gamma(2-\alpha)}{p^{1-\alpha}} \frac{\partial^{\alpha} H}{\partial p^{\alpha}}(d t)^{\alpha} .
\end{aligned}
$$

\section{Fractional Nambu Mechanics}

Method 1. In this part, we present the fractional Nambu mechanic based on fractional generalization of the form (3.6). As a result, we obtain

$$
\Omega_{\alpha}^{(2)}=q(d p)^{\alpha} \wedge(d r)^{\alpha}-H_{1}\left(d^{\alpha} H_{2}-(d t)^{\alpha}\right) .
$$

Taking

$$
\begin{gathered}
d^{\alpha}=\frac{\partial^{\alpha}}{\partial p^{\alpha}}(d p)^{\alpha}+\frac{\partial^{\alpha}}{\partial q^{\alpha}}(d q)^{\alpha} \\
\frac{\partial^{\alpha} q}{\partial q^{\alpha}}=\frac{q^{1-\alpha}}{\Gamma(2-\alpha)}
\end{gathered}
$$

the exterior of this form is

$$
\begin{aligned}
d^{\alpha} \Omega_{\alpha}^{(2)}= & \left(\frac{q^{1-\alpha}}{\Gamma(2-\alpha)}(d q)^{\alpha}-\frac{\partial^{\alpha}\left(H_{1}, H_{2}\right)}{\partial^{\alpha}(p, r)}(d t)^{\alpha}\right) \wedge\left((d p)^{\alpha}-\frac{\Gamma(2-\alpha)}{q^{1-\alpha}} \frac{\partial^{\alpha}\left(H_{1}, H_{2}\right)}{\partial^{\alpha}(q, r)}(d t)^{\alpha}\right) \\
& \wedge\left((d r)^{\alpha}-\frac{\Gamma(2-\alpha)}{q^{1-\alpha}} \frac{\partial^{\alpha}\left(H_{1}, H_{2}\right)}{\partial^{\alpha}(q, p)}(d t)^{\alpha}\right) .
\end{aligned}
$$

We lead to Pfaffian equations

$$
\begin{gathered}
\frac{q^{1-\alpha}}{\Gamma(2-\alpha)}(d q)^{\alpha}-\frac{\partial^{\alpha}\left(H_{1}, H_{2}\right)}{\partial^{\alpha}(p, r)}(d t)^{\alpha}=0 \\
(d p)^{\alpha}-\frac{\Gamma(2-\alpha)}{q^{1-\alpha}} \frac{\partial^{\alpha}\left(H_{1}, H_{2}\right)}{\partial^{\alpha}(q, r)}(d t)^{\alpha}=0 \\
(d r)^{\alpha}-\frac{\Gamma(2-\alpha)}{q^{1-\alpha}} \frac{\partial^{\alpha}\left(H_{1}, H_{2}\right)}{\partial^{\alpha}(q, p)}(d t)^{\alpha}=0 .
\end{gathered}
$$

It can be generalized as follows:

$$
\Omega_{\alpha}^{(n-1)}=x_{1}\left(d x_{2}\right)^{\alpha} \wedge \cdots \wedge\left(d x_{n}\right)^{\alpha}-H_{1} d^{\alpha} H_{2} \wedge \cdots \wedge d^{\alpha} H_{n-1} \wedge(d t)^{\alpha} .
$$


Method 2. In this part we present the fractional Nambu mechanics based on two Lagrangian formulation. In simple fractional Hamiltonian equations we have

$$
\begin{gathered}
{ }_{a} D_{t}^{\alpha} q=\frac{\partial H}{\partial p}=\frac{\partial(q, H)}{\partial(q, p)} \\
-{ }_{t} D_{b}^{\alpha} p=\frac{\partial H}{\partial q}=\frac{\partial(p, H)}{\partial(q, p)} .
\end{gathered}
$$

If we generalize these equations to Nambu fractional:

$$
\begin{aligned}
{ }_{a} D_{t}^{\alpha} q=\frac{\partial\left(q, H_{1}, H_{2}\right)}{\partial(q, p, r)}= & \left(\begin{array}{ccc}
\frac{\partial q}{\partial q} & 0 & 0 \\
\frac{\partial H_{1}}{\partial q} & \frac{\partial H_{1}}{\partial p} & \frac{\partial H_{1}}{\partial r} \\
\frac{\partial H_{2}}{\partial q} & \frac{\partial H_{2}}{\partial p} & \frac{\partial H_{2}}{\partial r}
\end{array}\right)=\frac{\partial H_{1}}{\partial p} \frac{\partial H_{2}}{\partial r}-\frac{\partial H_{2}}{\partial r} \frac{\partial H_{1}}{\partial p} \\
& -{ }_{t} D_{b}^{\alpha} p=\frac{\partial\left(p, H_{1}, H_{2}\right)}{\partial(q, p, r)}, \\
& -{ }_{t} D_{b}^{\alpha} r=\frac{\partial\left(r, H_{1}, H_{2}\right)}{\partial(q, p, r)} .
\end{aligned}
$$

In classical mechanics only variable, $q$, is considered as a configuration variables and variables, $p, r$ are first and second canonical variables, respectively. Suppose that it could be existed as many Lagrangian as the number of Hamiltonian. In the simplest case suppose that $L_{1}\left(q,{ }_{a} D_{t}^{\alpha} q\right)$ and $L_{2}\left(q,{ }_{a} D_{t}^{\alpha} q\right)$

$$
\begin{aligned}
& \delta \int L_{1}\left(q,{ }_{a} D_{t}^{\alpha} q\right) d t \\
& \delta \int L_{2}\left(q,{ }_{a} D_{t}^{\alpha} q\right) d t .
\end{aligned}
$$

Then the Fractional Euler-Lagrangian equations are

$$
\begin{aligned}
& \frac{\partial L_{1}}{\partial q}+{ }_{t} D_{b}^{\alpha} \frac{\partial L_{1}}{\partial_{a} D_{t}^{\alpha} q}=0, \\
& \frac{\partial L_{2}}{\partial q}+{ }_{t} D_{b}^{\alpha} \frac{\partial L_{2}}{\partial_{a} D_{t}^{\alpha} q}=0 .
\end{aligned}
$$


Next are defined the first and second canonical momentum $p, r$

$$
\begin{aligned}
& p=\frac{\partial L_{1}}{\partial_{a} D_{t}^{\alpha} q}, \\
& r=\frac{\partial L_{2}}{\partial_{a} D_{t}^{\alpha} q^{\prime}}
\end{aligned}
$$

and then the fractional Euler-Lagrange equations are

$$
\begin{aligned}
& { }_{t} D_{b}^{\alpha} p=\frac{\partial L_{1}}{\partial q}, \\
& { }_{t} D_{b}^{\alpha} r=\frac{\partial L_{2}}{\partial q} .
\end{aligned}
$$

Now we define two Hamiltonians $H_{1}$ and $H_{2}$ as follows:

$$
\frac{1}{{ }_{a} D_{t}^{\alpha} q} d\left[p_{a} D_{t}^{\alpha} q-L_{1}\right] \wedge d\left[r_{a} D_{t}^{\alpha} q-L_{2}\right]=d H_{1} \wedge d H_{2}
$$

Therefore we obtain

$$
{ }_{a} D_{t}^{\alpha} q d p \wedge d r+{ }_{t} D_{b}^{\alpha} p d r \wedge d q+{ }_{t} D_{b}^{\alpha} r d q \wedge d p=d H_{1} \wedge d H_{2}
$$

By expanding the right hand and by comparing the coefficients of form we lead to fractional Nambu mechanic equations.

This result can be generalized to the $n$-dimensional case, namely $L_{1}\left(q,{ }_{a} D_{t}^{\alpha} q\right)$, $\ldots, L_{n-1}\left(q,{ }_{a} D_{t}^{\alpha} q\right)$. As before we calculate the momenta $p_{1}=\partial L_{1} / \partial_{a} D_{t}^{\alpha} q, \ldots, p_{n}=\partial L_{(n-}$ 1) $/ \partial_{a} D_{t}^{\alpha} q$, and so on

$$
d H_{1} \wedge \cdots d H_{n-1}=\frac{1}{\left({ }_{a} D_{t}^{\alpha} q\right)^{n-2}} d\left(p_{1 a} D_{t}^{\alpha} q-L_{1}\right) \wedge \cdots d\left(p_{n-1 a} D_{t}^{\alpha} q-L_{n-1}\right)
$$

\section{A Fractional Lagrangian Formulation}

Let us assumed that the functions $q\left(t^{\prime}, t\right), p\left(t^{\prime}, t\right), r\left(t^{\prime}, t\right)$ are of two variable $t, t^{\prime}$. Then the corresponding Lagrangian is given by the following form:

$$
L=\int\left[q\left({ }_{t} D_{b}^{\alpha} p \frac{\partial r}{\partial t^{\prime}}-\frac{\partial p}{\partial t^{\prime}} t D_{b}^{\alpha} r\right)-H_{1}\left(\frac{\partial H_{2}}{\partial q} \frac{\partial q}{\partial t^{\prime}}+\frac{\partial H_{2}}{\partial p} \frac{\partial p}{\partial t^{\prime}}+\frac{\partial H_{2}}{\partial r} \frac{\partial r}{\partial t^{\prime}}\right)\right] d t^{\prime}
$$

Imposing

$$
\delta S=0,
$$


we obtain

$$
\begin{aligned}
\delta S=\iint[ & \delta q\left({ }_{t} D_{b}^{\alpha} p \frac{\partial r}{\partial t^{\prime}}-\frac{\partial p}{\partial t^{\prime}}{ }_{t} D_{b}^{\alpha} r\right)-\delta H_{1}\left(\frac{\partial H_{2}}{\partial q} \frac{\partial q}{\partial t^{\prime}}+\frac{\partial H_{2}}{\partial p} \frac{\partial p}{\partial t^{\prime}}+\frac{\partial H_{2}}{\partial r} \frac{\partial r}{\partial t^{\prime}}\right) \\
& \left.-H_{1} \delta\left(\frac{\partial H_{2}}{\partial q} \frac{\partial q}{\partial t^{\prime}}+\frac{\partial H_{2}}{\partial p} \frac{\partial p}{\partial t^{\prime}}+\frac{\partial H_{2}}{\partial r} \frac{\partial r}{\partial t^{\prime}}\right)\right] d t^{\prime} d t
\end{aligned}
$$

or

$$
\begin{aligned}
\delta S=\iint[ & \delta q\left(-\frac{\partial p}{\partial t^{\prime}} t D_{b}^{\alpha} r+{ }_{t} D_{b}^{\alpha} p \frac{\partial r}{\partial t^{\prime}}\right)-\frac{\partial H_{1}}{\partial q} \delta q\left(\frac{\partial H_{2}}{\partial q} \frac{\partial q}{\partial t^{\prime}}+\frac{\partial H_{2}}{\partial p} \frac{\partial p}{\partial t^{\prime}}+\frac{\partial H_{2}}{\partial r} \frac{\partial r}{\partial t^{\prime}}\right) \\
& \left.-H_{1}\left(\frac{\partial^{2} H_{2}}{\partial q^{2}} \delta q \frac{\partial q}{\partial t^{\prime}}+\frac{\partial H_{2}}{\partial q} \frac{\partial}{\partial t^{\prime}} \delta q+\frac{\partial^{2} H_{2}}{\partial p \partial q} \frac{\partial p}{\partial t^{\prime}} \delta q+\frac{\partial^{2} H_{2}}{\partial r \partial q} \frac{\partial r}{\partial t^{\prime}} \delta q\right)\right] d t^{\prime} d t .
\end{aligned}
$$

After some calculations, we obtain

$$
\begin{aligned}
\delta S=\iint\{ & {\left[\left({ }_{t} D_{b}^{\alpha} r-\frac{\partial\left(H_{1}, H_{2}\right)}{\partial(q, p)}\right) \frac{\partial p}{\partial t^{\prime}}-\left({ }_{t} D_{b}^{\alpha} p-\frac{\partial\left(H_{1}, H_{2}\right)}{\partial(r, q)}\right) \frac{\partial r}{\partial t^{\prime}}\right] \delta q } \\
+ & {\left[\left({ }_{a} D_{t}^{\alpha} q-\frac{\partial\left(H_{1}, H_{2}\right)}{\partial(p, r)}\right) \frac{\partial r}{\partial t^{\prime}}-\left({ }_{t} D_{b}^{\alpha} r-\frac{\partial\left(H_{1}, H_{2}\right)}{\partial(q, p)}\right) \frac{\partial q}{\partial t^{\prime}}\right] \delta p } \\
+ & {\left.\left[\left({ }_{t} D_{b}^{\alpha} p-\frac{\partial\left(H_{1}, H_{2}\right)}{\partial(r, q)}\right) \frac{\partial q}{\partial t^{\prime}}-\left({ }_{a} D_{t}^{\alpha} q-\frac{\partial\left(H_{1}, H_{2}\right)}{\partial(p, r)}\right) \frac{\partial p}{\partial t^{\prime}}\right] \delta r\right\} d t^{\prime} d t . }
\end{aligned}
$$

We conclude that if (5.7) is satisfied, then $\delta S=0$ for arbitrary variations of $\delta q, \delta p$ and $\delta r$.

\section{Conclusions}

In this paper, we defined new equations corresponding to the complex systems described by the Nambu mechanics within the languages of the fractional differential forms. It is shown that variation of the corresponding new action using fractional Lagrangian gives fractional Nambu equations. The equivalent methods presented in this manuscript can be applied to investigate the dynamics of the complex nonintegrable systems with memory. The classical results are obtained in the limiting case $\alpha \rightarrow 1$.

\section{References}

[1] J. Sabatier, O. P. Agrawal, and J. A. Tenreiro Machado, Advance in Fractional Calculus: Theoretical Developments and Applications in Physics and Engineering, Springer, Dordrecht, The Netherlands, 2007.

[2] D. Baleanu, B. Guvenc Ziya, and M. J. A. Tenreiro, New Trends in Nanotechnology and Fractional Calculus Applications, Springer, Dordrecht, The Netherlands, 2009.

[3] R. Hilfer, Applications of Fractional Calculus in Physics, World Scientific, Singapore, 2000.

[4] R. Gorenflo and F. Mainardi, Fractional Calculus: Integral and Differential Equations of Fractional Orders, Fractals and Fractional Calculus in Continuum Mechanics, Springer, New York, NY, USA, 1997. 
[5] A. A. Kilbas, H. M. Srivastava, and J. J. Trujillo, Theory and Applications of Fractional Differential Equations, vol. 204 of North-Holland Mathematics Studies, Elsevier, Amsterdam, The Netherlands, 2006.

[6] K. B. Oldham and J. Spanier, The Fractional Calculus, Academic Press, New York, NY, USA, 1974.

[7] K. S. Miller and B. Ross, An Introduction to the Fractional Integrals and Derivatives-Theory and Application, A Wiley-Interscience Publication, John Wiley \& Sons, New York, NY, USA, 1993.

[8] I. Podlubny, Fractional Differential Equations, vol. 198 of Mathematics in Science and Engineering, Academic Press, San Diego, Calif, USA, 1999.

[9] R. L. Magin, Fractional Calculus in Bioengineering, Begell House, Connecticut, Mass, USA, 2006.

[10] S. G. Samko, A. A. Kilbas, and O. I. Marichev, Fractional Integrals and Derivatives, Gordon and Breach, New York, NY, USA, 1993.

[11] B. J. West, M. Bologna, and P. Grigolini, Physics of Fractal Operators, Institute for Nonlinear Science, Springer, New York, NY, USA, 2003.

[12] G. M. Zaslavsky, Hamiltonian Chaos and Fractional Dynamics, Oxford University Press, Oxford, UK, 2005.

[13] F. Riewe, “Nonconservative Lagrangian and Hamiltonian mechanics," Physical Review E, vol. 53, no. 2, pp. 1890-1899, 1996.

[14] F. Riewe, "Mechanics with fractional derivatives," Physical Review E, vol. 55, no. 3, pp. 3581-3592, 1997.

[15] A. K. Golmankhaneh, A. K. Golmankhaneh, D. Baleanu, and M. C. Baleanu, "Hamiltonian structure of fractional first order Lagrangian," International Journal of Theoretical Physics, vol. 49, no. 2, pp. 365 $375,2010$.

[16] O. P. Agrawal, "Formulation of Euler-Lagrange equations for fractional variational problems," Journal of Mathematical Analysis and Applications, vol. 272, no. 1, pp. 368-379, 2002.

[17] O. P. Agrawal, "Fractional variational calculus and the transversality conditions," Journal of Physics A, vol. 39, no. 33, pp. 10375-10384, 2006.

[18] D. Baleanu and S. I. Muslih, "Lagrangian formulation of classical fields within Riemann-Liouville fractional derivatives," Physica Scripta, vol. 72, no. 2-3, pp. 119-121, 2005.

[19] D. Baleanu and O. P. Agrawal, "Fractional Hamilton formalism within Caputo's derivative," Czechoslovak Journal of Physics, vol. 56, no. 10-11, pp. 1087-1092, 2006.

[20] D. Baleanu, S. I. Muslih, and K. Taş, "Fractional Hamiltonian analysis of higher order derivatives systems," Journal of Mathematical Physics, vol. 47, no. 10, Article ID 103503, p. 8, 2006.

[21] D. Baleanu, A. K. Golmankhaneh, and A. K. Golmankhaneh, "Fractional Nambu mechanics," International Journal of Theoretical Physics, vol. 48, no. 4, pp. 1044-1052, 2009.

[22] G. S. F. Frederico and D. F. M. Torres, "A formulation of Noether's theorem for fractional problems of the calculus of variations," Journal of Mathematical Analysis and Applications, vol. 334, no. 2, pp. 834-846, 2007.

[23] A. K. Golmankhaneh, "Fractional poisson bracket," Turkish Journal of Physics, vol. 32, no. 5, pp. 241250, 2008.

[24] M. Klimek, "Fractional sequential mechanics-models with symmetric fractional derivative," Czechoslovak Journal of Physics, vol. 51, no. 12, pp. 1348-1354, 2001.

[25] M. Klimek, "Lagrangean and Hamiltonian fractional sequential mechanics," Czechoslovak Journal of Physics, vol. 52, no. 11, pp. 1247-1253, 2002.

[26] N. Laskin, "Fractional quantum mechanics," Physical Review E, vol. 62, no. 3 A, pp. 3135-3145, 2000.

[27] N. Laskin, "Fractional Schrödinger equation," Physical Review E, vol. 66, no. 5, Article ID 056108, 7 pages, 2002.

[28] S. I. Muslih and D. Baleanu, "Hamiltonian formulation of systems with linear velocities within Riemann-Liouville fractional derivatives," Journal of Mathematical Analysis and Applications, vol. 304, no. 2, pp. 599-606, 2005.

[29] E. M. Rabei, K. I. Nawafleh, R. S. Hijjawi, S. I. Muslih, and D. Baleanu, “The Hamilton formalism with fractional derivatives," Journal of Mathematical Analysis and Applications, vol. 327, no. 2, pp. 891-897, 2007.

[30] E. M. Rabei, D. M. Tarawneh, S. I. Muslih, and D. Baleanu, "Heisenberg's equations of motion with fractional derivatives," Journal of Vibration and Control, vol. 13, no. 9-10, pp. 1239-1247, 2007.

[31] E. M. Rabei, I. Almayteh, S. I. Muslih, and D. Baleanu, "Hamilton-Jacobi formulation of systems within Caputo's fractional derivative," Physica Scripta, vol. 77, no. 1, Article ID 015101, 2008.

[32] V. E. Tarasov, "Fractional variations for dynamical systems: Hamilton and Lagrange approaches," Journal of Physics A, vol. 39, no. 26, pp. 8409-8425, 2006. 
[33] V. E. Tarasov, "Fractional generalization of gradient and Hamiltonian systems," Journal of Physics A, vol. 38, no. 26, pp. 5929-5943, 2005.

[34] V. E. Tarasov, "Fractional vector calculus and fractional Maxwell's equations," Annals of Physics, vol. 323, no. 11, pp. 2756-2778, 2008.

[35] G. M. Zaslavsky, "Chaos, fractional kinetics, and anomalous transport," Physics Reports A, vol. 371, no. 6, pp. 461-580, 2002.

[36] D. Baleanu, A. K. Golmankhaneh, R. Nigmatullin, and A. K. Golmankhaneh, "Fractional Newtonian mechanics," Central European Journal of Physics, vol. 8, no. 1, pp. 120-125, 2010.

[37] A. K. Golmankahneh, A. K. Golmankahneh, and D. Baleanu, “On nonlinear fractional Klein Gordab equation," Signal Processing, vol. 91, no. 3, pp. 466-451, 2010.

[38] D. Baleanu, A. K. Golmankhaneh, and A. K. Golmankhaneh, "The dual action of fractional multi time Hamilton equations," International Journal of Theoretical Physics, vol. 48, no. 9, pp. 2558-2569, 2009.

[39] Y. Nambu, "Generalized Hamiltonian dynamics," Physical Review D, vol. 7, pp. 2405-2412, 1973.

[40] N. Mukunda and E. C. G. Sudarshan, "Relation between Nambu and Hamiltonian mechanics," Physical Review D, vol. 13, no. 10, pp. 2846-2850, 1976.

[41] L. Takhtajan, "On foundation of the generalized Nambu mechanics," Communications in Mathematical Physics, vol. 160, no. 2, pp. 295-315, 1994.

[42] M. Fecko, "On geometrical formulation of the Nambu dynamics," Journal of Mathematical Physics, vol. 33, no. 3, pp. 926-929, 1992.

[43] A. Cayley, Collected Mathematical Papers, Cambridge University Press, Cambridge, UK, 1890.

[44] H. Flanders, Differential Forms with Applicatons to the Physics Sciences, Dover Books on Advanced Mathematics, Dover, New York, NY, USA, 2nd edition, 1989.

[45] C. Shepherd and M. Naber, "Fractional differential forms II," http:/ /arxiv.org/abs/math-ph/0301016 (

[46] K. Cottrill-Shepherd and M. Naber, "Fractional differential forms," Journal of Mathematical Physics, vol. 42, no. 5, pp. 2203-2212, 2001. 